\title{
A Grazing-Incidence Small-Angle X-Ray Scattering View of Vertically Aligned ZnO Nanowires
}

\author{
M. Lučić Lavčević, ${ }^{1}$ P. Dubček, ${ }^{2}$ S. Bernstorff, ${ }^{3}$ M. Pavlović, ${ }^{2}$ and L. Šilovićc \\ ${ }^{1}$ Department of Physics, Faculty of Chemistry and Technology, University of Split, Teslina 10, 21000 Split, Croatia \\ ${ }^{2}$ Division of Materials Physics, Division of Laser and Atomic RぬD, Ruđer Bošković Institute, Bijenička 54, 10000 Zagreb, Croatia \\ ${ }^{3}$ SAXS Beamline, Elettra-Sincrotrone Trieste S.C.p.A., Strada Statale 14-km 163, 5 in AREA Science Park, 34149 Basovizza, Italy \\ Correspondence should be addressed to M. Lučić Lavčević; malula@ktf-split.hr
}

Received 3 August 2012; Revised 24 December 2012; Accepted 21 January 2013

Academic Editor: Claude Estournès

Copyright (C) 2013 M. Lučić Lavčević et al. This is an open access article distributed under the Creative Commons Attribution License, which permits unrestricted use, distribution, and reproduction in any medium, provided the original work is properly cited.

\begin{abstract}
We report a grazing-incidence small-angle $\mathrm{X}$-ray scattering study of $\mathrm{ZnO}$ films with vertically aligned and randomly distributed nanowires, grown through a hydrothermal growth process on nanostructured $\mathrm{ZnO}$ seeding coatings and deposited by electron beam evaporation on silicon and glass, respectively. The comparison of the scattering patterns of seeding coatings and nanowires showed that the scattering of vertically aligned nanowires exhibited a specific feature: the dominant characteristic of their scattering patterns is the appearance of fine structure effects around the specular peak. These effects were clarified by the combined reflection and scattering phenomena, suggested for the aligned nanowires-substrate system. Furthermore, they enabled the calculation of the average gyration radius of nanowires in horizontal direction. The calculated value was in good agreement with the radii of nanowires estimated by surface electron microscopy. Therefore, the observed feature in the scattering pattern can serve as evidence of the aligned growth of nanowires.
\end{abstract}

\section{Introduction}

Nanostructured materials are of growing interest for basic research and with respect to their applications. New properties of nanowires, nanotubes, nanolayers, and nanosheets, as examples of this class of materials, are based on surface effects and a size reduction to a regime in which the typical length scale of the nanostructure becomes comparable with a characteristic length scale of a physical phenomenon. Therefore, the control of morphology, which comprises size, shape, and crystallographic structure, plays a crucial role in the research and application of nanostructured materials, depending also on the progress in the characterization techniques.

The insight in the morphology of nanostructures is commonly realized by monitoring in the real space with electron microscopy (EM). Statistically averaged reciprocal space information, complementary to that obtained by EM, is provided by conventional X-ray diffraction (XRD) techniques and, in some cases, by synchrotron radiation-based nondestructive X-ray scattering techniques: small-angle Xray scattering (SAXS), wide-angle X-ray scattering (WAXS), and X-ray reflectivity (XR). For the SAXS and WAXS, analysis of bulk samples transmission geometry is usually used in scattering experiments. On the other hand, samples in form of thin films have to be examined in reflection geometry, that is, by grazing-incidence small-angle X-ray scattering (GISAXS) [1] and grazing-incidence wide-angle X-ray scattering (GIWAXS). Under the grazing incident conditions, the penetration of the $\mathrm{X}$-rays and, consequently, the examination of the selected sample area are ruled by choosing the angle of incidence. Therefore, not only the surface structures, but also the internal structures of thin films can be studied and the influence of substrate or the mixing of signals in composite or multilayered samples can be avoided. The ability of GISAXS method to get information about morphology of nanostructures in thin films depends on their distribution and organization. SAXS detects lengths on nanoscopic scales. The two-dimensional recording allows determination of dimensions of the scattering objects in the horizontal and vertical directions with respect to the film surface [2]. The eventual anisotropy of the measured scattering provides information regarding the shape and separation of nanostructures in film. 
Nanostructured systems which display ordering properties can be analyzed by means of determination of the characteristic lengths in both directions [3]. GIWAXS measurements are usually performed simultaneously with those of GISAXS, in order to collect data for the phase analysis. As large surface area is probed, GISAXS has a statistical significance compared to EM. Experiments are usually based on synchrotron radiation, due to the high demand on collimation.

In this paper, we focus on the GISAXS and GIWAXS measurements on $\mathrm{ZnO}$ nanostructured films with nanowires.

$\mathrm{ZnO}$ is a unique, key technological material that exhibits a wide range of useful properties. Numerous researches have been devoted to $\mathrm{ZnO}$ materials over the past decade.

$\mathrm{ZnO}$ is a wide band gap $(3.37 \mathrm{eV})$ semiconductor having a high electron-hole binding energy [4-6], which enables applications in electronics, optics, optoelectronics, lasers, and light-emitting diodes. For most of the currently advanced applications, $\mathrm{ZnO}$ is used in the form of thin film [7] or as a single crystal with a high degree of purity [8].

On the other hand, there is a rapid expansion in the research on $\mathrm{ZnO}$ as a nanostructured material. $\mathrm{ZnO}$ normally crystallizes in the wurtzite structure, characterized by the lack of a centre of symmetry and polar surfaces. The $\mathrm{ZnO}$ structure can be described by alternating planes with tetrahedrally coordinated $\mathrm{O}^{2-}$ and $\mathrm{Zn}^{2+}$ ions, stacked along the $c$-axis in an alternative manner. The electrostatic interaction energy and the chemical activity of the polar surfaces result in formation of numerous $\mathrm{ZnO}$ nanostructures. Due to size reduction, novel electrical, mechanical, chemical, and optical properties are introduced. Among nanostructures, nanowires [9] play an important role in developing nanoscience and nanotechnology: they are ideal for studying the one-dimensionally confined systems, important not only for understanding the fundamental phenomena, but also for developing applications. $\mathrm{ZnO}$ nanowires have shown great promise for application in nanometer scale photonic and light-emitting devices, in solar cells and in nanometer scale electronic applications, such as sensors or field emission transistors.

Beside various other techniques, $\mathrm{ZnO}$ nanowires elongated in the $c$-direction can be obtained through a slow, lowtemperature hydrothermal growth technique [10]. Although different preferential orientations of nanowires can be required in application, most of effort is focused toward directing them perpendicularly to the substrate ( $c$-axis orientation). A possible approach is to deposit a seed layer, on the substrate surface, on which the nanowires will be grown. Seeds can be introduced as dispersed nanograins, nanocrystalline coatings and single-crystalline substrates [11, 12].

We developed nanocrystalline seeding coatings of $\mathrm{ZnO}$, using the electron beam (e-beam) evaporation technique, to obtain $\mathrm{ZnO}$ nanowires through a seeded hydrothermal growth process from the commonly used precursors. The properties of coatings can be changed by the choice of substrate, deposition and annealing time, and temperature, which enable the growth of nanowires with different orientations.

For the presentation of GISAXS measurements performed on $\mathrm{ZnO}$ films, two representative samples, composed of substrates with seeding $\mathrm{ZnO}$ coatings and $\mathrm{ZnO}$ nanowires, were analyzed. First, nanowires, which were grown on a seeding coating deposited on silicon substrate, showed preferential orientation-they were aligned vertically on the substrate. Second, nanowires, which were grown on a seeding coating deposited on glass substrate, showed no imposed ordering - they were randomly oriented, mostly along the film surface.

Seeding coatings were standardly analyzed by GISAXS as systems of randomly distributed nanoparticles. Because of the statistical nature of the method, nanowires (as onedimensional micro-/nanostructures) are not expected to have a well-defined signature in the GISAXS pattern. This was confirmed for randomly oriented nanowires. On the contrary, the GISAXS patterns of vertically aligned nanowires showed a particular feature, which demonstrated their alignment.

GISAXS measurements were performed simultaneously with GIWAXS measurements, which enabled the insight in the crystallographic structure of coatings and nanowires.

Films were also examined in real space by the field emission scanning microscope (FE-SEM).

\section{Experimental}

2.1. Preparation of Samples. Two series of $\mathrm{ZnO}$ nanowires were grown on top of two different seeding coatings, obtained by using electron beam evaporation technique. Seeding coatings were deposited on silicon (100) and glass substrates.

The target used to fabricate coatings by electron beam technique was a high purity (99.99\%) polycrystalline $\mathrm{ZnO}$ source. The pressure of oxygen in the evaporation chamber was $9 \times 10^{-5}$ Torr and the temperature of substrates was $220^{\circ} \mathrm{C}$. The growth rate of films was $1 \AA \mathrm{s}^{-1}$. After the deposition, coated substrates were annealed in air for $5 \mathrm{~min}$.

The thickness of seeding coatings was measured using a Tencor-alpha-step profilometer and was found to be between $90 \mathrm{~nm}$ and $120 \mathrm{~nm}$.

For the growth of $\mathrm{ZnO}$ nanowires on coated substrates, a low-temperature chemical bath deposition was used [10]. The bath was equimolar solution of $1 \mathrm{mM} \mathrm{Zn}\left(\mathrm{NO}_{3}\right)_{2}$ and $\mathrm{C}_{6} \mathrm{H}_{12} \mathrm{~N}_{4}$ and only the side to be coated was immersed in the bath facing downwards. Nanowires were growing 12 hours in a laboratory oven, kept at the temperature of $85^{\circ} \mathrm{C}$.

At given preparation conditions, coatings on silicon enabled the growth of vertically aligned nanowires. Nanowires grown on coated glass substrates were randomly oriented. Samples chosen for the presentation of measurements performed on aligned and randomly distributed nanowires were a $100 \mathrm{~nm}$ thick seeding coating on silicon (for convenience denoted as $\mathrm{C} 1$ ), a $100 \mathrm{~nm}$ thick seeding coating on glass (denoted as C2), a nanowire film grown on coating $\mathrm{C1}$ (denoted as W1), and a nanowire film grown on coating C2 (denoted as W2).

2.2. Characterization. The insight in the general morphology of coatings and nanowires was realized by the electron microscope (FE-SEM Jeol 7100).

Further analysis of the nanosized objects was based on the simultaneous GISAXS/GIWAXS measurements at the SAXS 


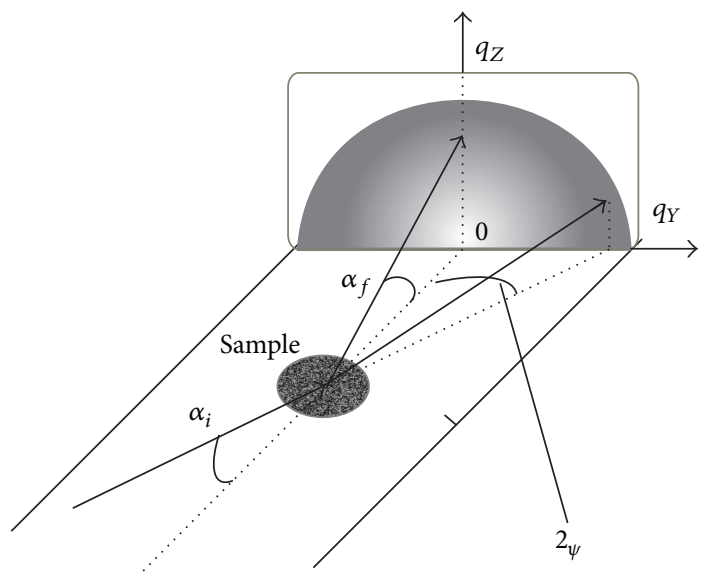

Figure 1: The geometry of GISAXS measurements: $\alpha_{i}$ and $\alpha_{f}$ are the incident and the exit angle of the X-ray beam, respectively, $2 \psi$ is the scattering angle in the horizontal plane, and $q_{Y}$ and $q_{Z}$ are the horizontal and the vertical scattering vectors, respectively.

beamline of the ELETTRA synchrotron radiation source [13]. A photon energy of $8 \mathrm{keV}(\lambda=0.154 \mathrm{~nm})$ was used, and the beam size was $4 \mathrm{~mm} \times 0.150 \mathrm{~mm}$ (horizontal $\times$ vertical). The measurements were performed at a grazing incident angle, $\alpha_{i}$, larger than the critical angle (the angle for total external reflection), to enable $\mathrm{X}$-rays to penetrate the sample. The critical angle, $\alpha_{C}$, which is is around $0.37^{\circ}$ for crystalline $\mathrm{ZnO}$, was determined experimentally for each film [14]. In the used setup for GISAXS/GIWAXS measurements, samples were mounted on a stepping-motor-controlled tilting stage with a step resolution of $0.001^{\circ}$. At first, the stage (and the sample surface) was aligned horizontally and parallel to the incoming beam within $\pm 0.1^{\circ}$. Then the incident angle was increased to $\alpha_{i}=\alpha_{C}+0.1^{\circ}$ and $\alpha_{i}=\alpha_{C}+0.3^{\circ}$, for $C$-samples and $W$-samples, respectively. The configuration for GISAXS measurement is illustrated in Figure 1.

The GISAXS intensity patterns were recorded by a twodimensional charge-coupled device (2D CCD) detector, containing $1024 \times 1024$ pixels and positioned perpendicular to the incident beam. A thin $\mathrm{Al}$ strip was placed in front of the detector to avoid its overflow in the specular plane direction. Two main directions, horizontal (in-plane) and vertical (out-ofplane), are represented by the position vectors in reciprocal space, that is, by the scattering vectors $q_{Y}$ and $q_{Z}$, respectively. Their moduli are defined by the wavelength $\lambda$, the incidence and exit angles $\alpha_{i}$ and $\alpha_{f}$, and the in-plane scattering angle $2 \psi$ of X-rays:

$$
\begin{gathered}
q_{Y}=2 \pi \lambda^{-1} \cos \alpha_{f} \sin \psi, \\
q_{Z}=2 \pi \lambda^{-1}\left(\sin \alpha_{i}+\sin \alpha_{f}\right) .
\end{gathered}
$$

Before numerical analysis, the GISAXS/GIWAXS patterns were corrected for readout noise and detector response.

GIWAXS measurements were performed simultaneously in the reflectivity plain using a position sensitive detector (PSD) and covering the angular range (2 $\theta$ ) from $25^{\circ}$ to $45^{\circ}$.
Phase identification was realized through matching diffracted peaks with PDF cards.

\section{Results and Discussion}

3.1. Coated Substrate. The morphologies of the coatings $\mathrm{Cl}$ and C2, analyzed by FE-SEM, are shown in Figures 2(a) and 2(b).

The images show that both silicon and glass plates are covered by particles of similar (nanometer) size and surface density. However, nanoparticles grown over glass plate are, on average, bigger (with majority of particles having diameters between 15 and $40 \mathrm{~nm}$ ) and of somewhat higher surface density than those on silicon substrate (where the majority of particles have diameters between 10 and $30 \mathrm{~nm}$ ).

Figure 3 shows the GIWAXS patterns of coatings $\mathrm{C} 1$ and $\mathrm{C} 2$. We can see that the coatings had the pure wurtzite structure. GIWAXS pattern of $\mathrm{C} 1$ has (002) as dominant reflection, while that of C2 shows (100), (002), and (101) reflections. The crystallinity of the films is better in $\mathrm{Cl}$.

GIWAXS cannot be used for a straightforward interpretation of intensity scans with respect to a preferred crystallographic orientation in $\mathrm{C} 1$ and $\mathrm{C} 2$. The incident angle is fixed in a grazing incidence and the scan normal varies with the diffraction angle and, therefore, each diffraction peak corresponds to a different set and orientation of the reflecting planes. Since the geometry of measurements is not symmetrical, some reflections can be hindered. However, some assumptions about orientation can be made. As shown in Figure 3(b), all dominant peaks appeared in the GIWAXS patterns of the coating $\mathrm{C} 2$, indicating that the crystallites are probably randomly oriented or only weakly textured. For an accurate estimate of the texture, conventional XRD measurements are needed. Because of the strong absorption in silicon and glass, the XRD measurements that we performed in Bragg-Brentano geometry showed (002) reflection as the only resoluble peak in the pattern of the coating $\mathrm{Cl}$, while no peaks were recorded for the coating C2. Together with the result of GIWAXS, this indicates that a significant portion of the crystallites in $\mathrm{Cl}$ is oriented with their $c$-axis parallel or slightly tilted with respect to the substrate surface normal.

The average crystallite sizes calculated from the profile width at half maximum height of the given peaks in GIWAXS patterns, using the Scherrer equation [15] with included instrumental line broadening, were around $13 \mathrm{~nm}$ for $\mathrm{Cl}$ and around $7 \mathrm{~nm}$ for $\mathrm{C} 2$. Estimate by XRD for $\mathrm{C} 1$ indicates a value of $20 \mathrm{~nm}$. It can be concluded that particles (as seen by FE-SEM) in $\mathrm{Cl}$ are mostly either single crystalline or composed of only few crystallites, while those in C2 are either polycrystalline or present agglomeration of undifferentiated smaller particles.

The 2D GISAXS patterns of coatings (C1 and $\mathrm{C} 2)$ are shown in Figures 4(a) and 4(b), respectively. The scattering patterns represent the maps of scattered intensities in the given in-plane and out-of-plane angular scattering ranges. The maximum of the scattered intensity is in the direction of the specular plane $\left(q_{Y}=0\right)$, which is normal to the surface of the sample. This scattered intensity is partly reduced by a thin $\mathrm{Al}$ absorber (vertical strips in the patterns are depleted 


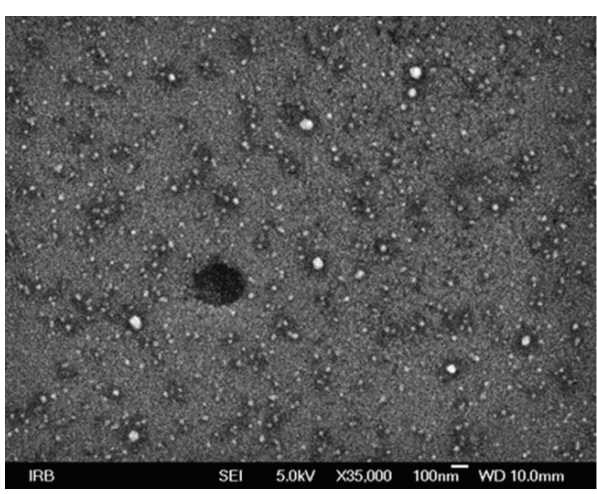

(a)

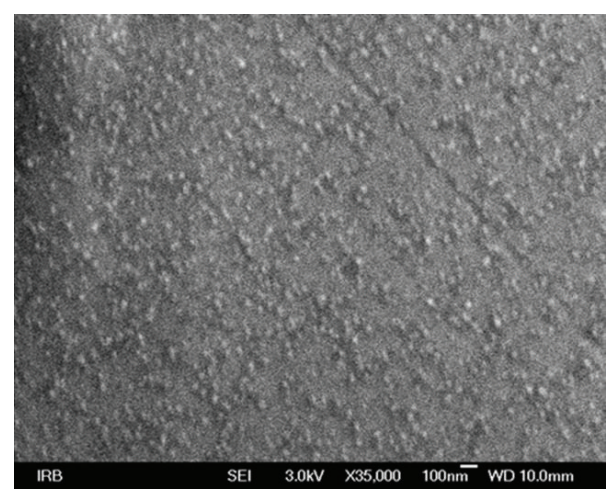

(b)

FIGURE 2: FE-SEM images of the ZnO coatings: (a) C1, on silicon, and (b) C2, on glass. Figures are recorded with 35000 magnification and $5 \mathrm{kV}$ electron beam.

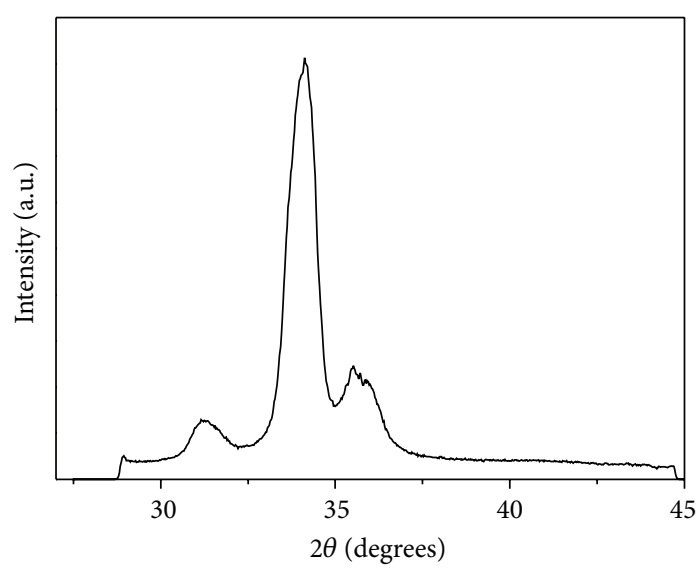

(a)

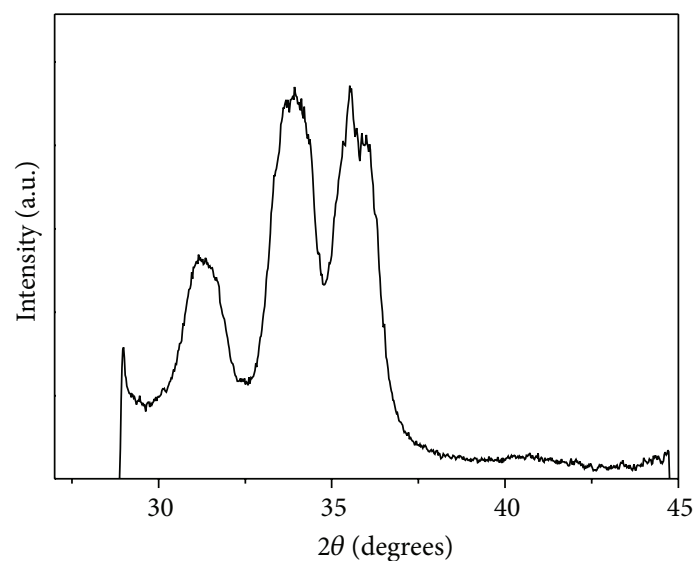

(b)

FIgURE 3: GIWAXS patterns of the ZnO coatings: (a) C1, on silicon, and (b) C2, on glass.

intensities). For exit angles equal to incident critical angle $\left(\alpha_{f}=\alpha_{C}\right)$, there is an enhancement in the scattering intensity due to the refraction effects, which is also known as Yoneda peak.

2D GISAXS patterns allow determination of dimensions of these scattering objects along the two main directions, horizontal (parallel to the sample surface) and vertical (parallel to the specular plane), represented by the scattering vectors $q_{Y}$ and $q_{z}$. The method of interpreting scattering data is based on the analysis of the intensity cuts, which shows the dependence of the intensity, $I$, on the scattering vector, $q_{i}\left(q_{Y}\right.$ or $\left.q_{Z}\right)$. The intensity cuts, that is, one-dimensional scattering curves, taken from Figure 4, at the fixed values of horizontal and vertical scattering vectors (relatively close to the Yoneda peak and the specular plane, resp.), were subjected to the Guinier analysis of the average particle size.

Generally, scattering depends on the form factor of the scattering object, which is the absolute squared Fourier transform of the object shape function. In the Guinier approximation [16], the central part of the form factor has a unique shape. The scattering in the small $q$-region depends only on the overall size of the object and the scattered intensity can be expressed as

$$
I(q) \propto \exp \left(\frac{-R_{G}^{2} q^{2}}{3}\right),
$$

where $R_{G}$ is the so-called Guinier or gyration radius that represents the average (effective) size of the scattering objects and can be related to the dimensions of objects of various shapes.

The gyration radii, $R_{G Y}$ and $R_{G Z}$, which give information about the average scattering object size in the vertical and horizontal directions, respectively, were calculated by fitting the corresponding $1 \mathrm{D}$ intensity cuts, in the $L n I$ versus $q^{2}$ presentation, for the region of very small scattering vectors. From a slope of the fitting line, the corresponding gyration radius is calculated as

$$
R_{G}=(-3 \beta)^{1 / 2} .
$$

This reduces to a Gaussian shape of the scattering intensity for very small angles, and the width of the Gaussian is inversely 


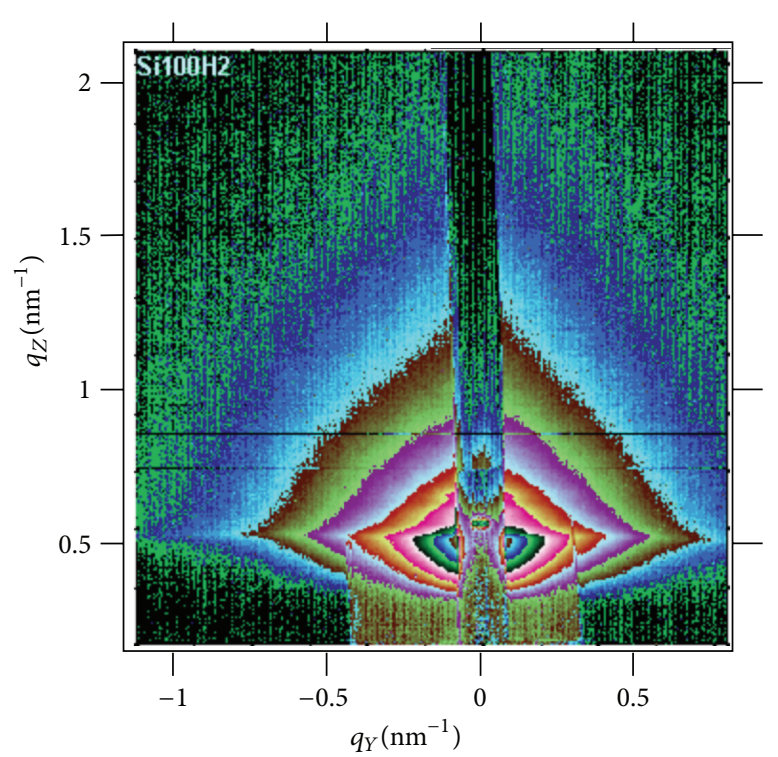

(a)

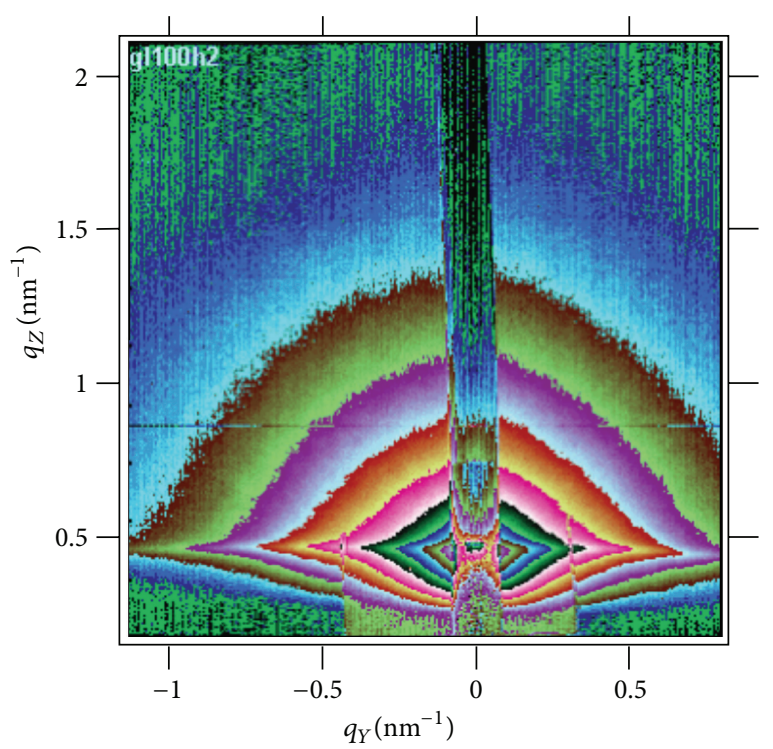

(b)

Figure 4: 2D GISAXS patterns of the $\mathrm{ZnO}$ coatings: (a) $\mathrm{C}$, on silicon, and (b) $\mathrm{C} 2$, on glass.

proportional to the scattering object size. The obtained sizes for the scattering objects, that is, particles in the coating $\mathrm{C} 1$, are $R_{G Y}=9 \mathrm{~nm}$ and $R_{G Z}=6 \mathrm{~nm}$ in horizontal and vertical direction, respectively. The difference between horizontal and vertical direction value suggests anisotropic shape of particles in this coating, which can be related to its texture. The values of the gyration radii for the coating $\mathrm{C} 2$ have similar values in both directions, $R_{G Y}=9 \mathrm{~nm}$ and $R_{G Z}=10 \mathrm{~nm}$, indicating particles of almost spherical shape. The results of the GISAXS analysis are consistent with the estimations made by FE-SEM.

3.2. Nanowires. The general morphologies of the nanowires in samples W1 and W2, analyzed by FE-SEM, are shown in Figures 5(a) and 5(b).

The images suggest that nanowires in sample W1 preferentially grow aligned in vertical direction, while those in W2 grow in a more random way. Since some of nanowires partly lie in the plane of the sample surface, it is obvious that their length is of the order of micrometer, as expected, considering the type of hydrothermal preparation, which causes them to elongate along the $c$-axis, forming highly anisotropic nanostructures. Their diameters are of the order of ten nanometers.

The GIWAXS measurements showed that $\mathrm{ZnO}$ is the only phase in the grown nanowires as demonstrated in Figures 6(a) and 6(b). The possible preferred orientation is indicated for the sample W1, as well as its absence for the sample W2.

Comparing these results with those of GIWAXS/XRD for $\mathrm{C} 1$ and $\mathrm{C} 2$, we can clearly see the preferential orientation of the nanowires structure as compared to the coatings.

The 2D GISAXS patterns of samples $\mathrm{W} 1$ and $\mathrm{W} 2$ are shown in Figures 7(a) and 7(b), respectively.

After the nanowires growth, GISAXS pattern changes significantly as can be seen from comparison of W1 (Figure 7(a)) to $\mathrm{C} 1$ (Figure $4(\mathrm{a})$ ). The main feature of the scattering pattern of W1 is a well-separated asymmetric distribution of scattered intensities around the specular peak (Figure 7(c)). In order to analyze this pattern, we assume a model of nanowires standing upright on the substrate, as shown in Figure 8 that illustrates the scattering in the specular plane. Since the film structure is porous, there are three distinct contributions to the scattered intensity.

(i) Direct beam is scattered.

(ii) Direct beam is reflected and then scattered.

(iii) Direct beam is scattered, and scattered beam is reflected.

Relative intensities of these three contributions are hard to asses without a precise insight into substrate surface coverage (or relative fraction of the area which contributes to the reflection) and local roughness values which additionally reduce the reflection coefficient. Nevertheless, the lower side of the angular range of the first type of contribution is limited at least by the angle of total reflection due to the refraction effects, which sets the top limit on the size of the particles that can be evaluated. Therefore, the scattering at very small angles (scattering objects are rather large) from the first contribution is depleted in the sample. Only the scattering at somewhat larger angles (smaller particles) prevails. However, both the direct beam and the scattered signal are reflected from substrate surface, and the intensity is given by the coefficient of reflection, which steeply decays with increasing angle. Therefore, the other two contributions give scattering signal that is centered around the reflected beam and their intensity is a function of the reflection angle. Here, we neglect the higher order effects, like scattering on the substrate surface itself, which are typically much smaller when the substrate surface is rather smooth. 

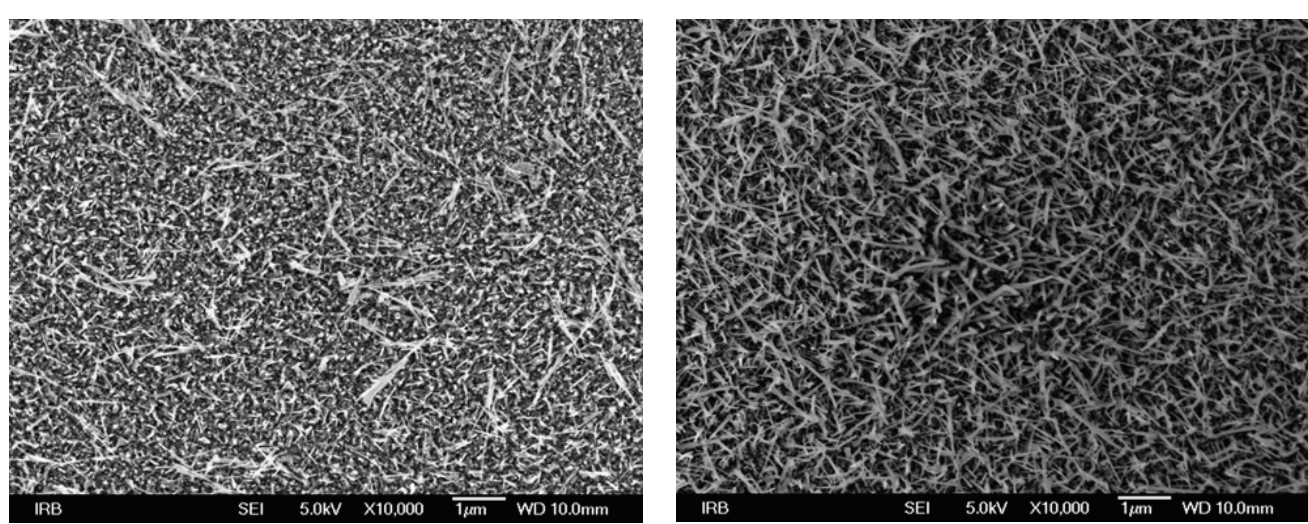

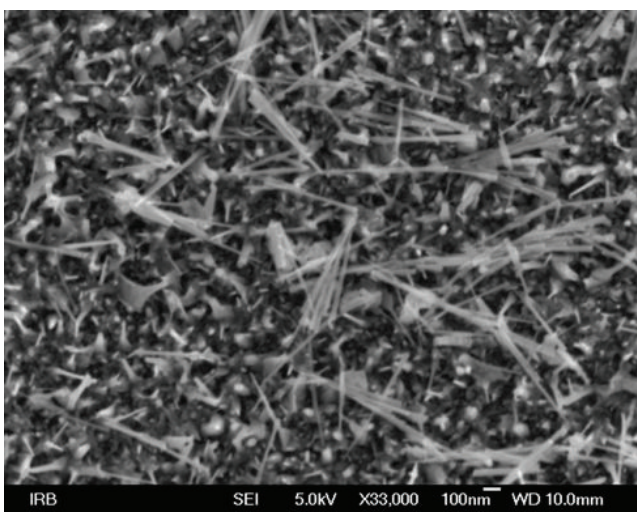

(a)

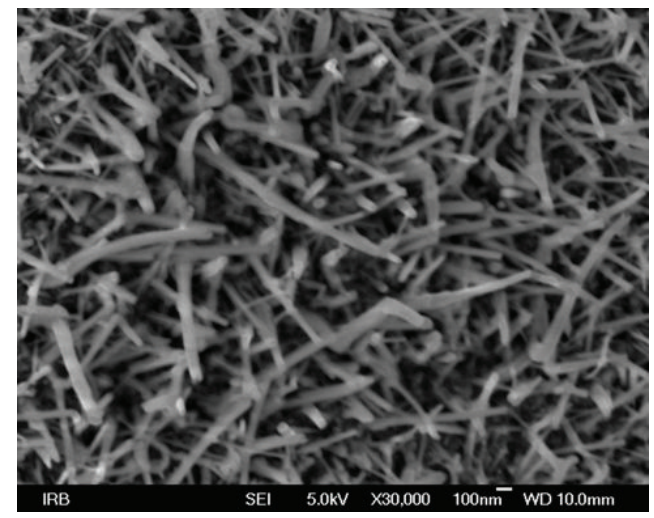

(b)

FiguRE 5: FE-SEM images of the ZnO nanowires: (a) W1, on coated silicon, and (b) W2, on coated glass. Top figures (a) and (b) are recorded with 10000 magnification and $5 \mathrm{kV}$ electron beam. Bottom figures (a) and (b) are recorded with 33000 and 30000 magnification, respectively.

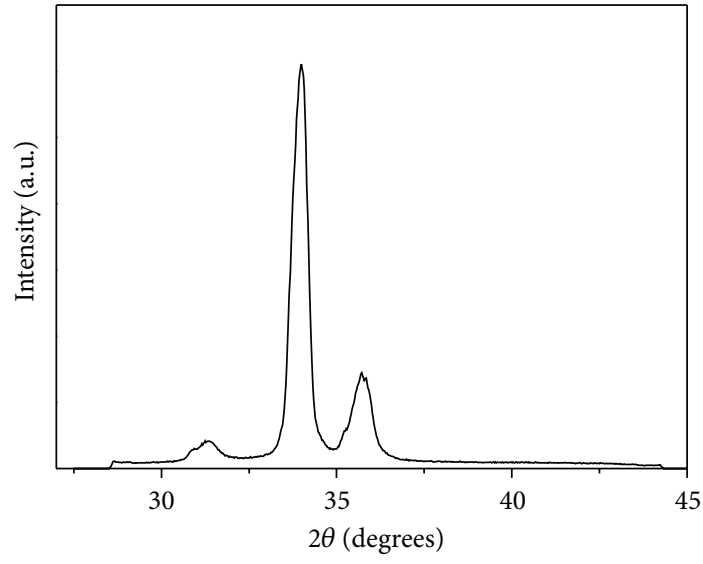

(a)

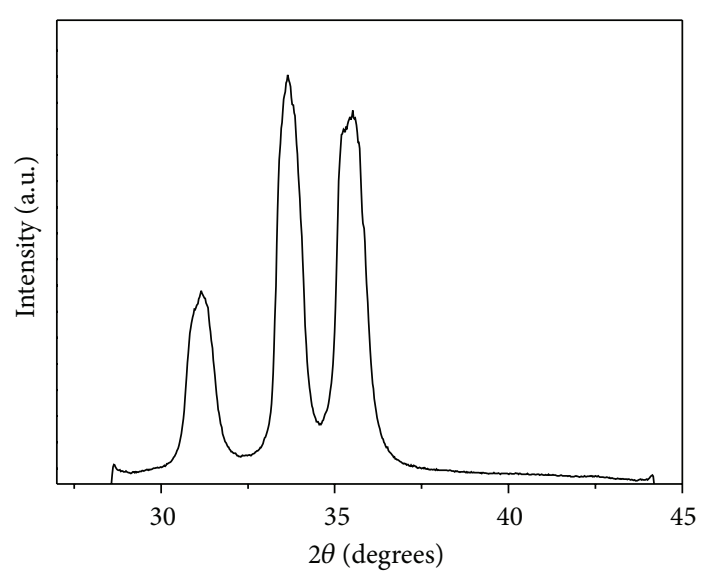

(b)

FIgURE 6: GIWAXS patterns of the $\mathrm{ZnO}$ nanowires: (a) W1, on coated silicon, and (b) W2, on coated glass.

In the analysis of the reflected signals, we have to take account of the reflection directions. For the sake of illustration in Figure 8, we restricted the scattering process to the specular plain. In this plain, there are two extreme cases: in the case of scattering away from reflecting plane, the angle of reflection is $(\alpha-\omega)$, where $\alpha$ is the angle of specular reflection and $\omega$ is the scattering angle. In the opposite case, the angle of reflection is $(\alpha+\omega)$. Therefore, the scattered intensity is modulated by reflection. As the scattering intensity is proportional to the form factor of scattering objects, the amplitude of the form factor, FF, has to be weighted by the corresponding coefficient of reflection $(R(\alpha), R(\alpha-\omega)$, or $R(\alpha+\omega))$ to account for the reflection effects. For the given angle of reflection in the specular plain, the scattering in the direction perpendicular to this plain is not a function of the horizontal scattering angle. 


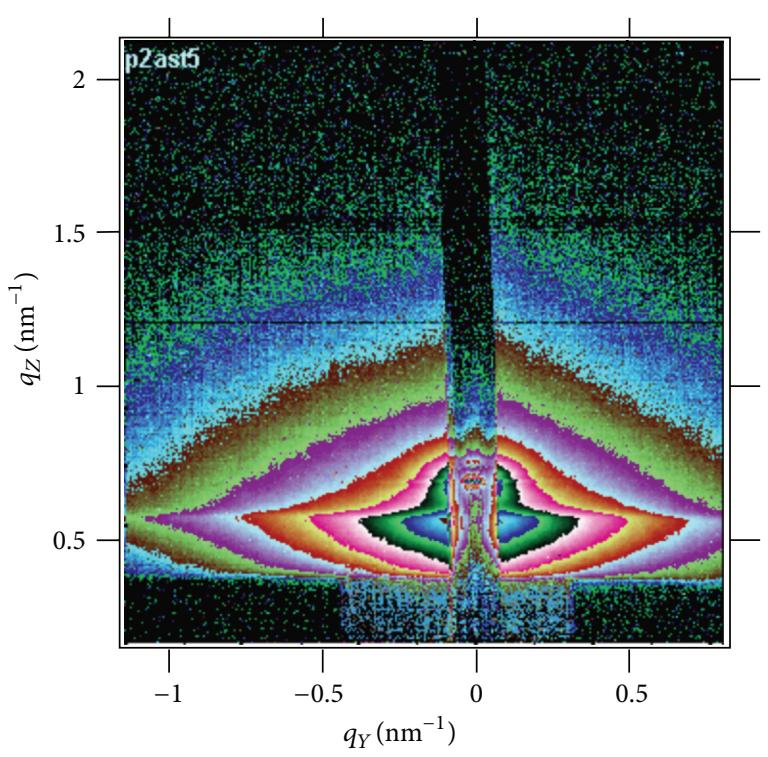

(a)

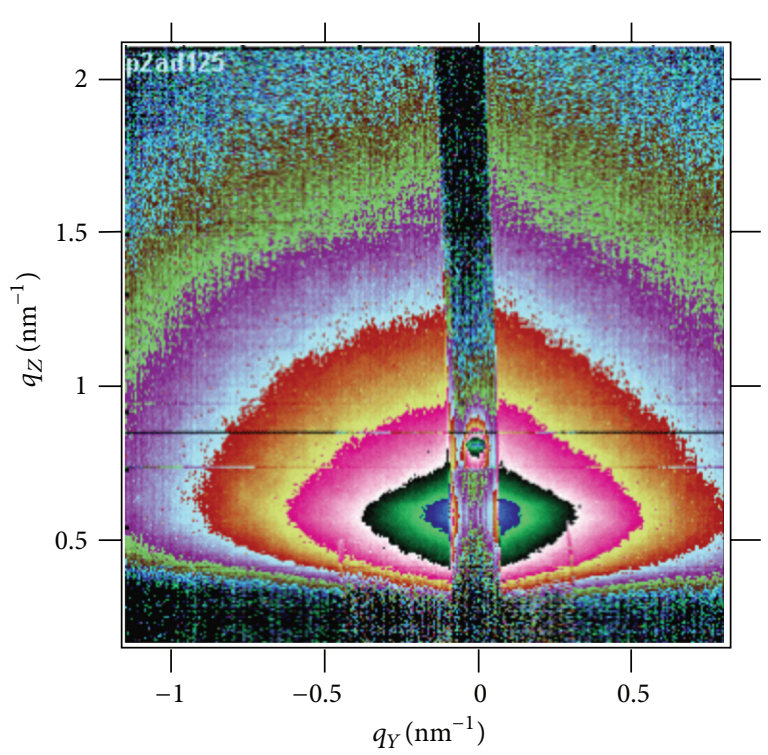

(b)

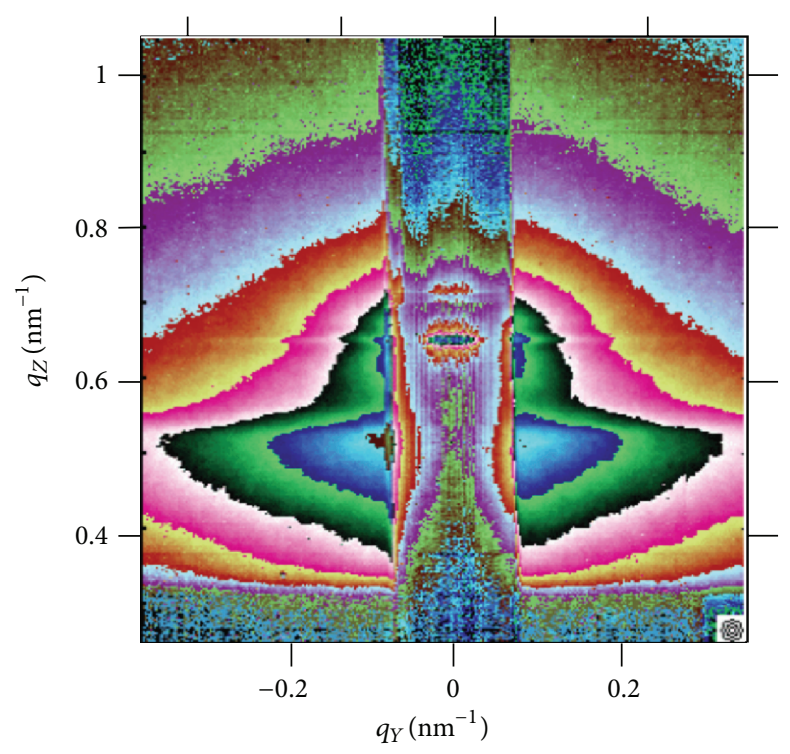

(c)

FIGURE 7: 2D GISAXS patterns of samples with ZnO nanowires: (a) W1 and (b) W2; (c) is zoomed in part of (a) centered around the specular peak.

Therefore, the contribution around the specular peak has been subject to numerical analysis in two directions: intensities along horizontal $\left(q_{Z}=0.7 \mathrm{~nm}^{-1}\right)$ and vertical $\left(q_{Y}=\right.$ $0.1 \mathrm{~nm}^{-1}$ ) directions were used in the Guinier analysis. The vertical cut is illustrated in Figure 9. The obtained sizes are $R_{G Y}=15 \mathrm{~nm}$ and $R_{G Z}=22 \mathrm{~nm}$ in horizontal and vertical direction, respectively. This is in good agreement with FESEM results: the radii of nanowires correlate well with the values obtained for radii of gyration. Keeping in mind that the Guinier radius is effectively sensitive only on the particle cross-section in the direction perpendicular to the cut to which it is applied and the FE-SEM results, we ascribe the difference between horizontal and vertical direction value to preferred orientation of the nanowires, which is apparent in FE-SEM images too.

In case of the sample W2, the described scattering effect in the specular plane is not visible. This sample has been grown on the glass substrate, which has initially bigger roughness than monocrystalline silicon, and this results in substantially weaker reflected intensity. Bigger roughness probably favours bigger particle formation during electron beam evaporation. Therefore, the GISAXS from C2 is stronger than that of C1 (see Figure 4). This initial roughness results in less ordered growth of nanowires and it is evident in less defined GISAXS pattern. Namely, scattering from W2 has poorly defined Yoneda peak at $q_{Z}=0.6 \mathrm{~nm}^{-1}$. This results from 


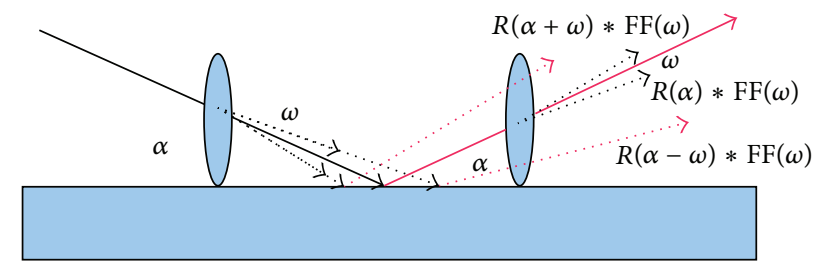

FIGURE 8: Illustration of scattering in the specular plane. $\alpha$ is the angle of incidence and specular reflection, $\omega$ is the scattering angle, FF is amplitude of the form factor, and $R$ is the coefficient of reflection for a given angle.

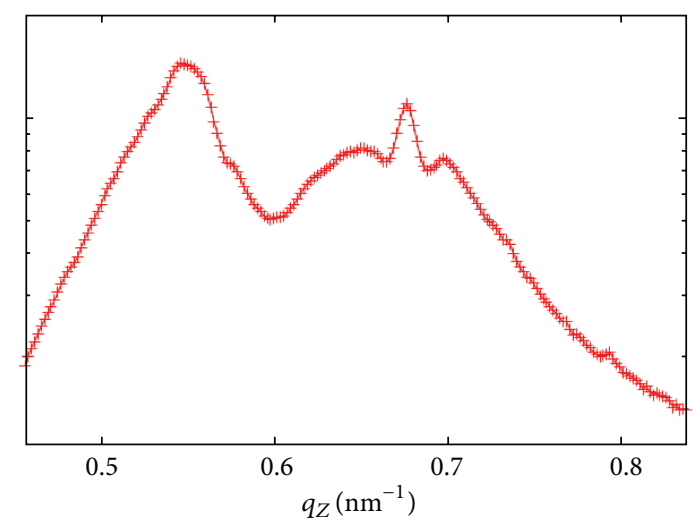

FIGURE 9: Vertical cut of the scattered intensity, extracted from Figure 7(a) for sample W1.

significant surface roughness on the scale much larger than the wavelength used. Finally, as we see from the FE-SEM image in Figure 5(b), the nanowires in this sample are not isotropically oriented, but are set to cover most of the substrate surface. Consequently, scattering is dominated by the contribution that does not include reflection on the surface (i.e., by the above-mentioned first contribution to the scattered intensity).

The numerical analysis of the scattering contributions results in the Guinier radii averaged over all nanowire orientations, and also those parallel to the substrate are present. Final hindrance is the limitation posed by the cutoff at critical angle, which reduces detectable sizes to about $10 \mathrm{~nm}$. The obtained Guinier radius value would be misleading.

The general results shown for representative samples of $\mathrm{ZnO}$ nanowires, grown on $100 \mathrm{~nm}$ thick $\mathrm{ZnO}$ seeding coatings on silicon and glass, maintained consistency for all samples of both series. Typical gyration radii, with values between $14 \mathrm{~nm}$ and $16 \mathrm{~nm}$ in horizontal direction, were estimated for the samples of aligned nanowires grown on coated silicon.

Moreover, the preliminary GISAXS measurements (not shown here) performed on vertically aligned $\mathrm{ZnO}$ nanowires, grown on the flat but thicker $\mathrm{ZnO}$ seeding coatings and under different growth conditions, clearly showed the described fine structure effect in the scattering patterns, which can be used for the estimation of the gyration radii.

\section{Conclusion}

In summary, different nanocrystalline seeding coatings of $\mathrm{ZnO}$, with thicknesses between 90 and $120 \mathrm{~nm}$, were obtained on silicon and glass, using the electron beam evaporation technique in order to enable the growth of nanowires with different orientations. At that range of thicknesses, the coatings deposited on silicon promoted the growth of vertically aligned nanowires, while the coatings on glass substrates enabled only the growth of nanowires with almost random orientation. The offered explanation is that initially bigger roughness of glass as compared to monocrystalline silicon results in more disordered $\mathrm{ZnO}$ coating deposition, and this itself results in significantly less oriented nanowires.

GISAXS measurements on vertically aligned nanowires exhibited a fine structure effect around the specular peak. This was explained by the model, which assumes a combination of reflection and scattering phenomena in nanowiressubstrate systems. Specifically, in a system of aligned nanowires, the considerable surface area of the substrate is undisturbed by nanowires and therefore the reflection of $\mathrm{X}$ rays is significant. The numerical analysis of the distribution intensities around the specular peak enabled the estimation of the gyration radii of about $15 \mathrm{~nm}$ in the horizontal direction, which are in good agreement with the radii of nanowires estimated by FESEM analysis of samples.

Therefore, it can be concluded that the fine structure effect in the GISAXS pattern of vertically aligned nanowires on substrates reflects the specific geometry of the system and can serve as the evidence of their vertically ordered growth.

The measurements on nanowires grown on coated glass substrates support this conclusion: the described scattering effect in the specular plane is not visible. The GISAXS analysis of these almost randomly oriented nanowires does not provide a coherent result, because of the statistical nature of this method.

In our previous research, the statistical nature of GISAXS method enabled successful characterization of nanostructures randomly distributed in thin films, such as those in seeding coatings analyzed in this paper, producing information about films that may be critical in case of their further application. On the other hand, the same statistical nature should, generally, prevent the application of GISAXS in characterization of micro-/nanostructures, such as nanowires. However, our results demonstrate that the nanosized dimension of nanowires, standing upward on the substrate, can be assessed by using GISAXS. This new kind of GISAXS characterization of nanowires proves to be straightforward in case of partial coverage of a flat substrate, regaining information at the smallest angles that would not otherwise be available due to sample and substrate absorption below the critical angle.

Further investigation of vertically aligned nanowires, grown on substrates by different procedures, should test and complement this concept of characterization. As GISAXS measurements can be performed in various environments, we expect that the appearance of the described fine structure scattering effect should also be useful during in situ monitoring of the early stages of the nanowires growth and ordering. 


\section{Acknowledgment}

The results shown in this work are obtained in the frame of the scientific project "Nanostructures of metal oxides and metals: morphology and properties," supported by the Ministry of Science, Education and Sport of the Republic of Croatia.

\section{References}

[1] J. R. Levine, J. B. Cohen, Y. W. Chung, and P. Georgopoulos, "Grazing-incidence small-angle X-ray scattering: new tool for studying thin film growth," Journal of Applied Crystallography, vol. 22, pp. 528-532, 1989.

[2] M. Lučić Lavčević, P. Dubček, A. Turković, Z. Crnjak-Orel, and S. Bernstorff, "Nanostructural depth profile of vanadium/cerium oxide film as a host for lithium ions," Solar Energy Materials and Solar Cells, vol. 91, no. 7, pp. 616-620, 2007.

[3] M. Lučić Lavčević, A. Turković, P. Dubček, Z. Crnjak Orel, B. Orel, and S. Bernstorff, "GISAXS view of induced morphological changes in nanostructured $\mathrm{CeVO}_{4}$ thin films," Journal of Nanomaterials, vol. 2011, Article ID 303808, 7 pages, 2011.

[4] J. Wu and D. Xue, "Progress of science and technology of $\mathrm{ZnO}$ as advanced material," Science of Advanced Materials, vol. 3, no. 2, pp. 127-149, 2011.

[5] U. Özgür, Y. I. Alivov, C. Liu et al., "A comprehensive review of $\mathrm{ZnO}$ materials and devices," Journal of Applied Physics, vol. 98, no. 4, Article ID 041301, pp. 1-103, 2005.

[6] S. Singh, P. Thiyagarajan, K. Mohan Kant et al., "Structure, microstructure and physical properties of $\mathrm{ZnO}$ based materials in various forms: bulk, thin film and nano," Journal of Physics D, vol. 40, no. 20, pp. 6312-6327, 2007.

[7] E. Fortunato, A. Gonçalves, A. Pimentel et al., "Zinc oxide, a multifunctional material: from material to device applications," Applied Physics A, vol. 96, no. 1, pp. 197-205, 2009.

[8] A. Janotti and C. G. Van De Walle, "Fundamentals of zinc oxide as a semiconductor," Reports on Progress in Physics, vol. 72, no. 12, Article ID 126501, 2009.

[9] Z. L. Wang, "Splendid one-dimensional nanostructures of zinc oxide: a new nanomaterial family for nanotechnology," ACS NANO, vol. 2, no. 10, pp. 1987-1992, 2008.

[10] A. Sugunan, H. C. Warad, M. Boman, and J. Dutta, "Zinc oxide nanowires in chemical bath on seeded substrates: role of hexamine," Journal of Sol-Gel Science and Technology, vol. 39, no. 1, pp. 49-56, 2006.

[11] S. Yamabi and H. Imai, "Growth conditions for wurtzite zinc oxide films in aqueous solutions," Journal of Materials Chemistry, vol. 12, no. 12, pp. 3773-3778, 2002.

[12] T. Kawano, J. Yahiro, H. Maki, and H. Imai, "Epitaxial growth of wurtzite $\mathrm{ZnO}$ crystals in an aqueous solution system," Chemistry Letters, vol. 35, no. 4, pp. 442-443, 2006.

[13] H. Amenitsch, S. Bernstorff, and P. Laggner, "High-flux beamline for small-angle X-ray scattering at ELETTRA," Review of Scientific Instruments, vol. 66, no. 2, pp. 1624-1626, 1995.

[14] P. Dubček, "Nanostructure as seen by the SAXS," Vacuum, vol. 80, no. 1-3, pp. 92-97, 2005.

[15] B. D. Cullity and S. R. Stock, Elements of X-Ray Diffraction, Prentice Hall, New York, NY, USA, 2001.

[16] A. Guinier, "La diffraction des rayons $\mathrm{X}$ aux tres petits angles; application á l'etude de phenomenes ultramicroscopiques," Annals of Physics, vol. 12, pp. 161-237, 1939. 

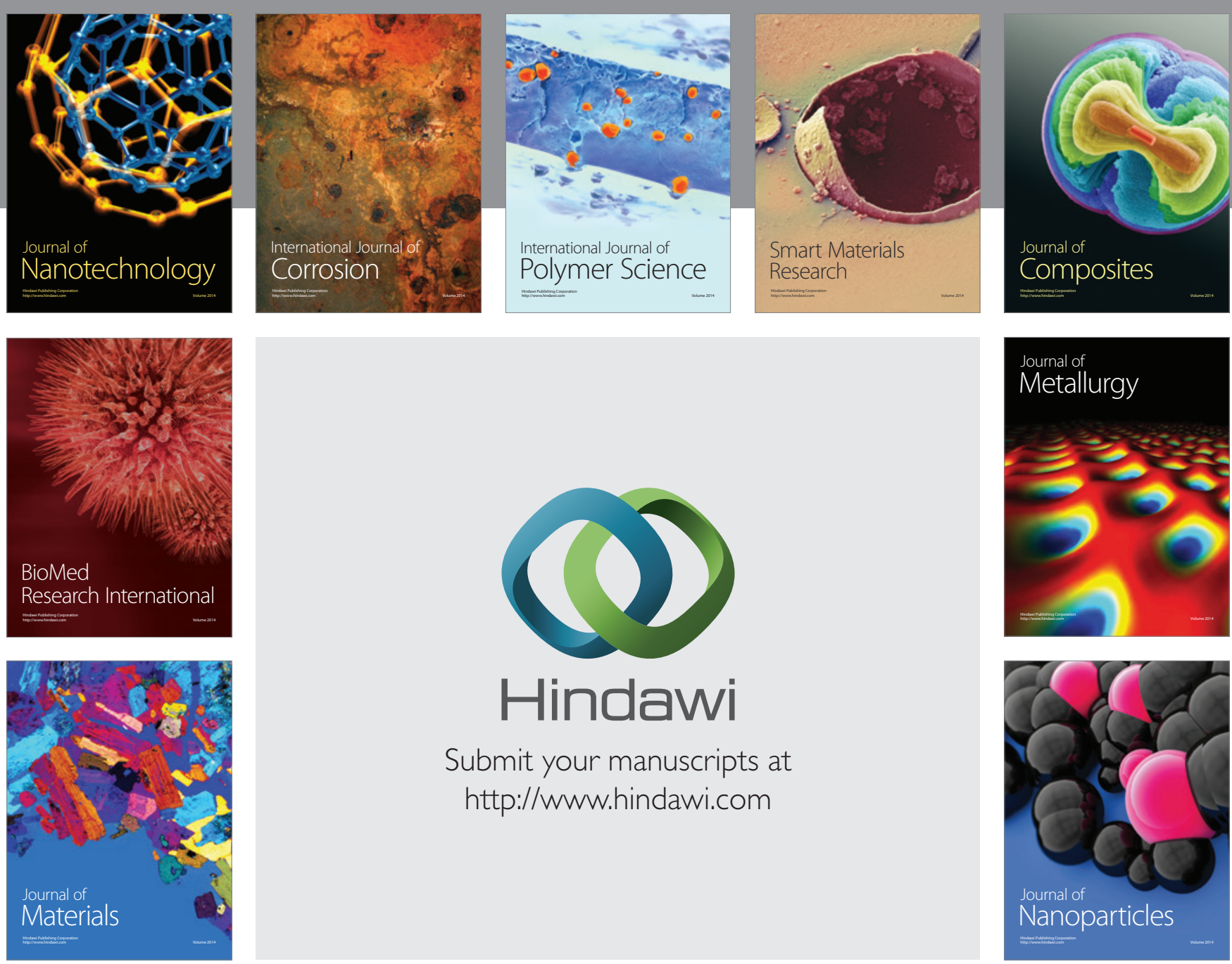

Submit your manuscripts at http://www.hindawi.com
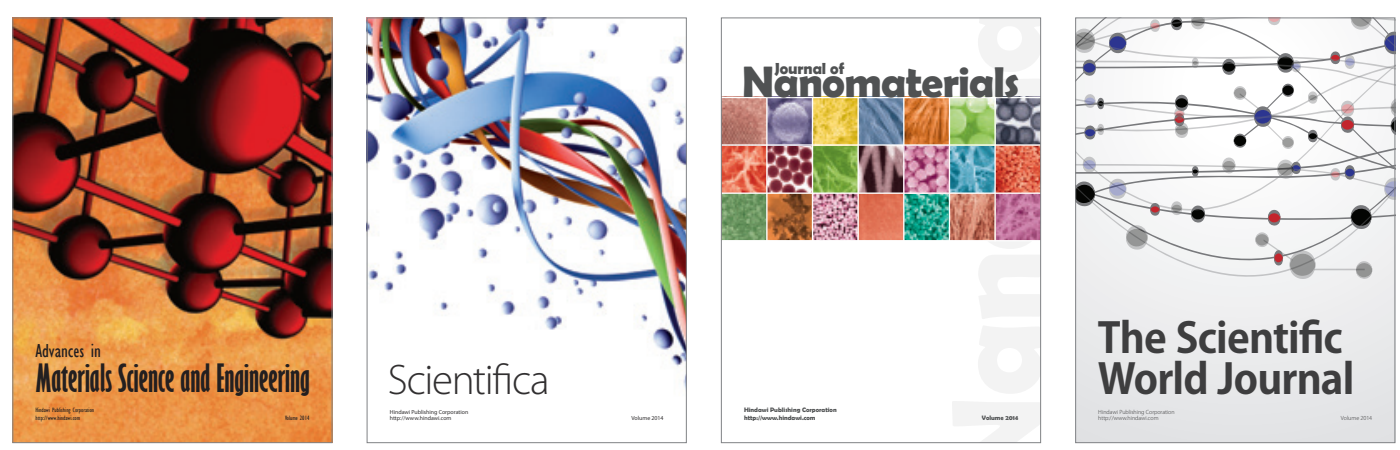

\section{The Scientific World Journal}
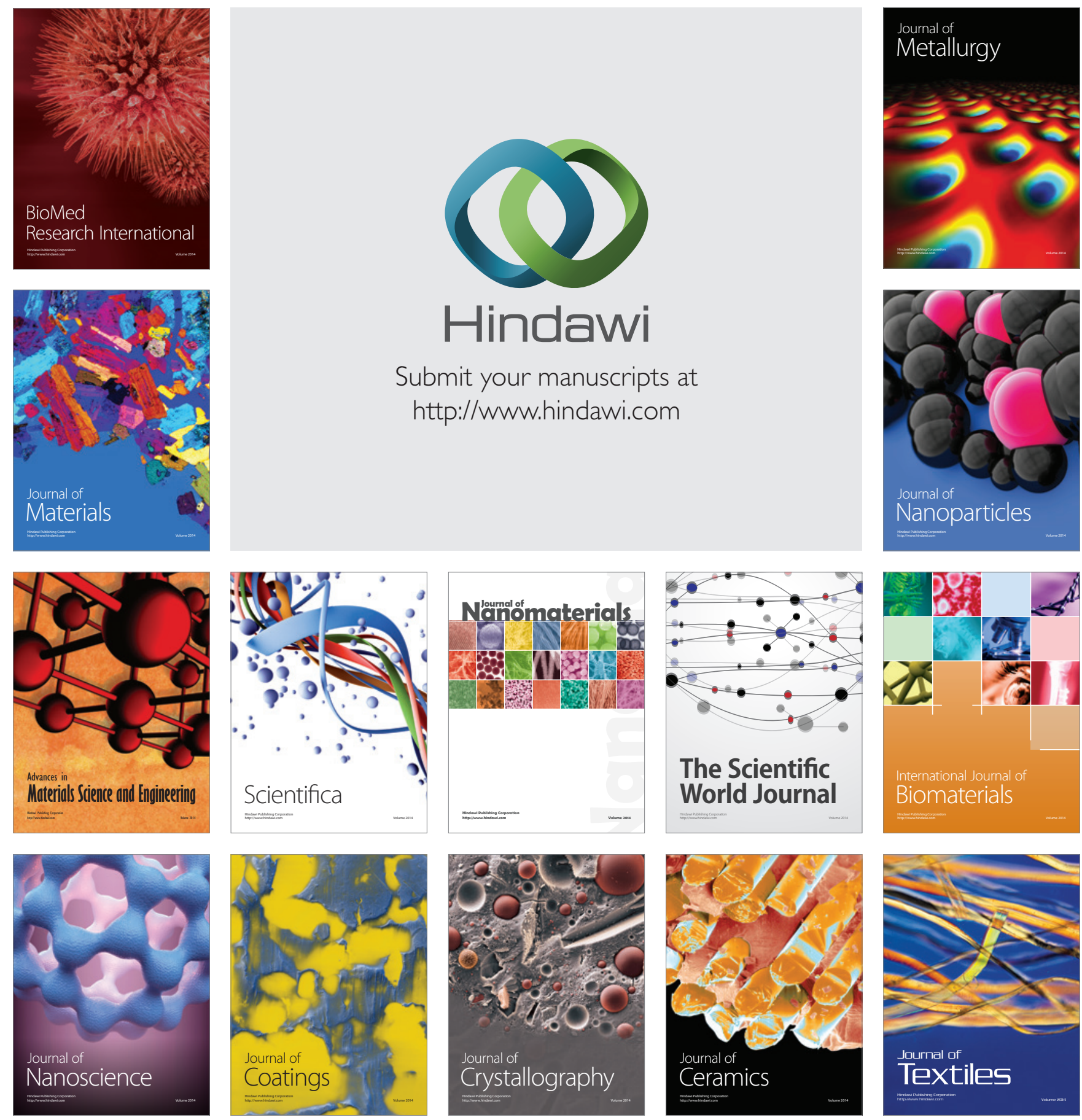